\title{
Experiência de sucesso na implantação de leitos de unidade de terapia intensiva adulta no Norte de Minas Gerais
}

\author{
Elisabete Costa Reis Dutra \\ Graduação em Farmácia-Bioquímica pela Universidade Federal de Juiz de Fora com habilitação em Análises Clínicas \\ (1987). Atualmente é Assessora Chefe da Assessoria de Contratos Assistenciais da Secretaria Estadual de Saúde de \\ Minas Gerais e Pós Graduanda do Mestrado Profissional de Inovação Biofarmacêutica da UFMG. \\ elisabete.costa@saude.mg.gov.br
}

\section{Helidéa de Oliveira Lima}

Médica pela Universidade Federal de Juiz de Fora e especialista em Gestão Hospitalar. atuação nas áreas de Planejamento Estratégico e Gestão da Qualidade. Subsecretária de Políticas e Ações de Saúde da Secretaria de Estado de Saúde de Minas Gerais

helidea@saude.mg.gov.br

\section{Jacqueline Salles Diniz \\ ja.diniz@yahoo.com.br}

\section{Osvaldo Kurschus de Oliveira}

Possui graduação em Ciências Contábeis pela Pontifícia Universidade Católica de Minas Gerais (1986) e graduação em Administração de Empresa pela Pontifícia Universidade Católica de Minas Gerais (1987). Atualmente membro do Observatório de Custos em Saúde da Secretaria de Estado de Saúde de Minas Gerais - SES/MG.

osvaldo.oliveira@saude.mg.gov.br

\section{Renata Melgaço Gonçalves}

renata.melgaço@saude.mg.gov.br

\section{Telma Braga Orsini}

Graduada em Fisioterapia pela Faculdade de Ciências Médicas de Minas Gerais,(1980) e Especialista em Economia da Saúde pela Escola de Governo Professor Paulo Neves de Carvalho - Fundação João Pinheiro :MG (2006). Atualmente é Coordenadora do Núcleo de Suporte e Informação da Superintendência de Assistência Farmacêutica - Secretaria de Estado de Saúde de MG.

telma.braga@saude.mg.gov.br

RESUMO: Este artigo objetiva relatar experiência exitosa na implementação da Rede de Urgência e Emergência (UeE) com foco nos leitos de Unidade de Terapia Intensiva (UTI), na Macrorregião Norte, do Estado de Minas Gerais, no período de 2002 a 2010, nos hospitais públicos previamente selecionados, pertencentes as microrregiões pólos de Brasília de Minas, Janaúba, Pirapora, e Taiobeiras. O planejamento do trabalho desenvolvido nesse relato, na escolha dessas micros teve como indicadores de saúde escolhidos pela Coordenadoria de UTI da Secretaria de Estado de Minas Gerais (SES/MG): Índice de Desenvolvimento Humano (IDH), Disability Adjusted Life Years (DALY) ou Anos de Vida Perdidos Ajustados por Incapacidade (AVPI), Deficiência dos Hospitais, Localização Geográfica, Renda Per Capta, Tripla Carga de Doenças, Alto Índice de Mortalidade, e outros. Tendo como resultados a ampliação do parque de leitos de UTI no total de 40 leitos nesses municípios, onde foram criadas câmaras técnicas de trauma, cardiologia e AVC, diminuição da mortalidade, e núcleos de educação permanente implantados.

PALAVRAS-CHAVE: Leitos de Unidade de Terapia Intensiva. Macrorregião Norte de Minas Gerais. Rede Urgência e Emergência.

\section{Successful experience in the implementation of adult intensive care unit in the north of Minas Gerais}

ABSTRACT: This article aims at reporting successful experience in the implementation of Urgent and Emergency Network (UUT) with focus on the beds in the Intensive Care Unit (ICU), in Macroregion North of Minas Gerais in the period 2002 to 2010 in public hospitals previously selected, belonging to the micro clusters Brasilia de Minas, Janaúba, Pirapora and Taiobeiras. The planning of work in this story, in choosing these micros had as health indicators chosen by the Coordination of ITU Secretary of State of Minas Gerais (SES / MG): Human Development Index (HDI), Disability Adjusted Life Years (DALY) or life years Disability-Adjusted (AVPI), Disability, Hospital Geographic Location, per capita income, Triple Burden of Disease, high mortality, and others. Having as a result of expansion of the ICU beds in total 40 beds in these municipalities, where cameras were set up techniques of trauma, cardiology and stroke, decreased mortality, and continuing education centers deployed.

KEY WORDS: Beds in the Intensive Care Unit. Macro northern region of Minas Gerais. Urgent and Emergency Network. 


\section{INTRODUÇÃO}

Navegar pelas águas da administração dos parques de leitos das UTI da UeE da Macrorregião Norte de Minas Gerais, tem exigido de nós gestores e profissionais da SES/MG, esforços e compromissos redobrados em direção a uma ação planejada. Afinal, de modo equivalente aos desafios impostos pelos mares e oceanos, o terreno da administração de implantação de leitos de UTI da Rede de UeE nesta Macrorregião impõe intempéries e imprevistos, seja nos períodos de bonança seja diante da virada das marés.

Até o poeta lusitano Fernando Pessoa, admirável escritor da língua portuguesa evidencia no verso "navegar é preciso; viver não é preciso" o entendimento da necessidade de uma ação ordenada quando no momento da definição de rotas e caminhos. O termo "preciso" é apropriado na dimensão de "exatidão", não somente de "necessidade". O poeta sugere que a arte de navegar é uma ciência; já a vida é imprecisa e cercada de incertezas. E, para enfrentar a imprevisibilidade das marés, ou os riscos da vida, planejar é preciso.

Analogamente aos navegadores, nós, gestores e profissionais da saúde, encontramos inúmeros desafios e imprevistos ao longo dessa jornada e precisamos sempre estar aptos para enfrentá-los, assim, encontrando respostas adequadas para os problemas encontrados. Aprendemos que o planejamento não é uma ferramenta que suprime ou constrói desvios a fim de evitar quadros indesejáveis ou crises, ao contrário, ele aponta, assim como uma bússola, as rotas e caminhos a seguir, para o enfretamento e a superação das adversidades, considerando a escassez de recursos e a minimização de impactos da região estudada.

Utilizamos o aprendizado de todo o esforço do governo de MG de enfrentar o equilíbrio das contas públicas e de por na agenda a necessidade de uma gestão pública eficiente, medidas adotadas a partir de $2003 \mathrm{com}$ o choque de gestão, aportam, já em 2007, no caminho da ordenação de prioridades e do planejamento, ou, em outras palavras, nos resultados. E tão importante quanto à definição da rota estabelecida pelo Plano Mineiro de Desenvolvimento Integrado (PMDI), 2007-2023, que são os mecanismos de correção e aperfeiçoamento. Ou seja, é agir estrategicamente adotando instrumentos que permitam a adoção de melhorias e a correção das imperfeições.

Esse foco nos orientou, assim como as cartas e instrumentos de navegação, o caminho a seguir para uma gestão eficiente e eficaz na implementação de leitos de UTI - UeE nos hospitais públicos previamente selecionados, pertencentes aos municípios de Brasília de Minas, Janaúba, Pirapora, e Taiobeiras desta carente Macrorregião do Norte de MG, mostrada na figura 2, e empenhados na adoção de políticas públicas comprometidas com a melhoria de vida desta população.

Figura 1: municípios da Macro Norte estudados/Macrorregião Pólo

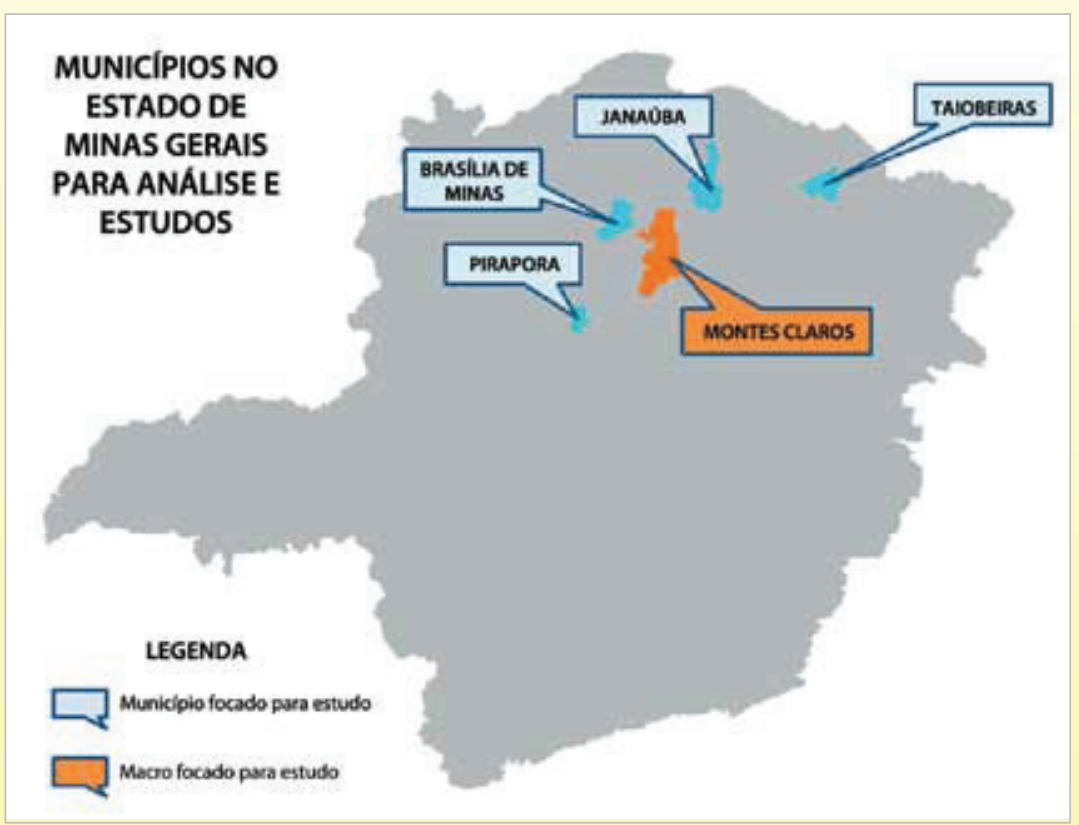

Fonte: PDR/ MG /2009 - Deliberação CIB-SUS/mg nº 618 de 09 de dezembro de 2009 
O planejamento do trabalho desenvolvido nesse relato teve como indicadores selecionados pela Coordenadoria de UTI da SES/MG: IDH, DALY, Deficiência dos Hospitais, Localização Geográfica, Renda Per Capta, Tripla Carga de Doenças, - Alto Índice de Mortalidade, e outros.

Apresentamos a seguir o percurso metodológico ao navegarmos nesse estudo tendo por meta além de salvar vidas, proporcionar uma vida saudável a essa população usuária do Sistema Único de Saúde (SUS), reconhecendo que a partir de 2007, a maturidade da gestão por resultados ficou sendo muito maior do que no seu início. Apresentamos esse estudo com a certeza de que mais um pilar de sustentação foi levantado para a construção, conhecimento e análise de um efetivo modelo de gestão por resultados,

\section{PERCURSO METODOLÓGICO}

Este estudo foi baseado na Rede de UeE com foco nos Leitos de UTI nos hospitais públicos selecionados da Macrorregião Norte da SES/MG.

\subsection{Características Gerais de Minas Gerais}

Minas Gerais é uma das 27 unidades federativas do Brasil, sendo a quarta maior em extensão territorial, com 586.528,3 km². Localiza-se no Sudeste e limita-se a sul e sudoeste com São Paulo, a oeste com o Mato Grosso do Sul e a noroeste com Goiás, incluindo uma pequena divisa com o Distrito Federal, a leste com Espírito Santo, a sudeste com o Rio de Janeiro e a norte e nordeste com a Bahia. O estado é o segundo mais populoso do Brasil, com pouco mais de 20 milhões de habitantes, e sua capital é a cidade de Belo Horizonte, que reúne em sua região metropolitana cerca de cinco milhões de habitantes.

O Instituto Brasileiro de Geografia e Estatística (IBGE) decompôs o estado em 12 mesorregiões ${ }^{1}$, que consistem em: - Campo das Vertentes, - Central Mineira, - Jequitinhonha, - Metropolitana de Belo Horizonte (BH), - Noroeste de Minas, - Norte de Minas, - Oeste de Minas, - Sul e Sudoeste de Minas, Triângulo Mineiro e Alto Paranaíba, - Vale do Mucuri, - Vale do Rio Doce, e Zona da Mata.

O Norte de Minas, região definida para este estudo, é a maior das mesorregiões e abrange 86 municípios (IBGE, 2006), ocupando uma área de aproximadamente de $128 \mathrm{mil} \mathrm{km}^{2}$ e abrigando um pouco mais de 1,5 milhões de habitantes (IBGE, estimativa 2005), sendo maior que os estados de Pernambuco ou Santa Catarina. Tem peculiaridades similares às da Região Nordeste do Brasil. O clima é quente, beirando o semi-árido, formado por Planalto Atlântico. A economia é baseada na pecuária e extrativismo vegetal.

MG possui o terceiro maior Produto_Interno_Bruto do Brasil (PIB), superado apenas pelos estados de São Paulo e Rio de Janeiro. Dados dos estudos realizados pelos pesquisadores Ralfo e Garcia, 2006, relativo a espacialidade do PIB mineiro tendo como foco somente as mesorregiões, os mesmos chegaram a situações referente ao ano de 2003 comparativo do PIB tendo a região metroplitana de Belo Horizonte atigido $42,4 \%$, a região Norte de 3,9 \% e região Noroeste de 1,7\%. O que evidência a desigualdade econômica no estado.

Para uma melhor gerência a SES/MG, partindo de um dos princípios organizativos do SUS, a regionalização, agrupou os municípios do estado em macro e microrregiões com o objetivo de melhor assegurar o acesso dos cidadãos a todas as ações necessárias à resolução de seus problemas de saúde proporcionando a otimização dos recursos disponíveis, a hierarquização do sistema, e a incrementar a resolução da maior parte dos agravos no nível primário de atenção. Cognominado como Plano Diretor de Regionalização (PDR), MG adota desde 2002, uma divisão territorial específica visando a melhor resposta sanitária a determinadas situações de saúde, contendo assim, 13 macrorregiões e 76 microrregiões.

\subsection{Características da Macrorregião Norte}

A Macrorregião Norte é composta por sete microrregiões de saúde. A região apresenta uma forte desigualdade social e baixa densidade demográfica de 11,9 Hab/ km2. As distâncias entre municípios são superiores a $500 \mathrm{~km}$ e há extensas áreas rurais com concentração populacional alta, comparada

\footnotetext{
é uma subdivisão dos estados brasileiros que congrega diversos municípios de uma área geográfica com similaridades econômicas e sociais. Foi criada pelo IBGE e é utilizada para fins estatísticos e não constitui, portanto, uma entidade ou administrativa. Wikipédia, 2010.
} 
às outras realidades no próprio estado de MG. Montes Claros desempenha a função de município pólo macrorregional e 12 municípios são pólos microrregionais, sendo eles: Brasília de Minas, Bocaiúva, Taiobeiras, Januária, Janaúba, Salinas, Monte Azul, Coração de Jesus, Pirapora, São Francisco, Francisco Sá, e Manga conforme Figura 2.

\section{Figura 2: micros da macrorregião Norte}

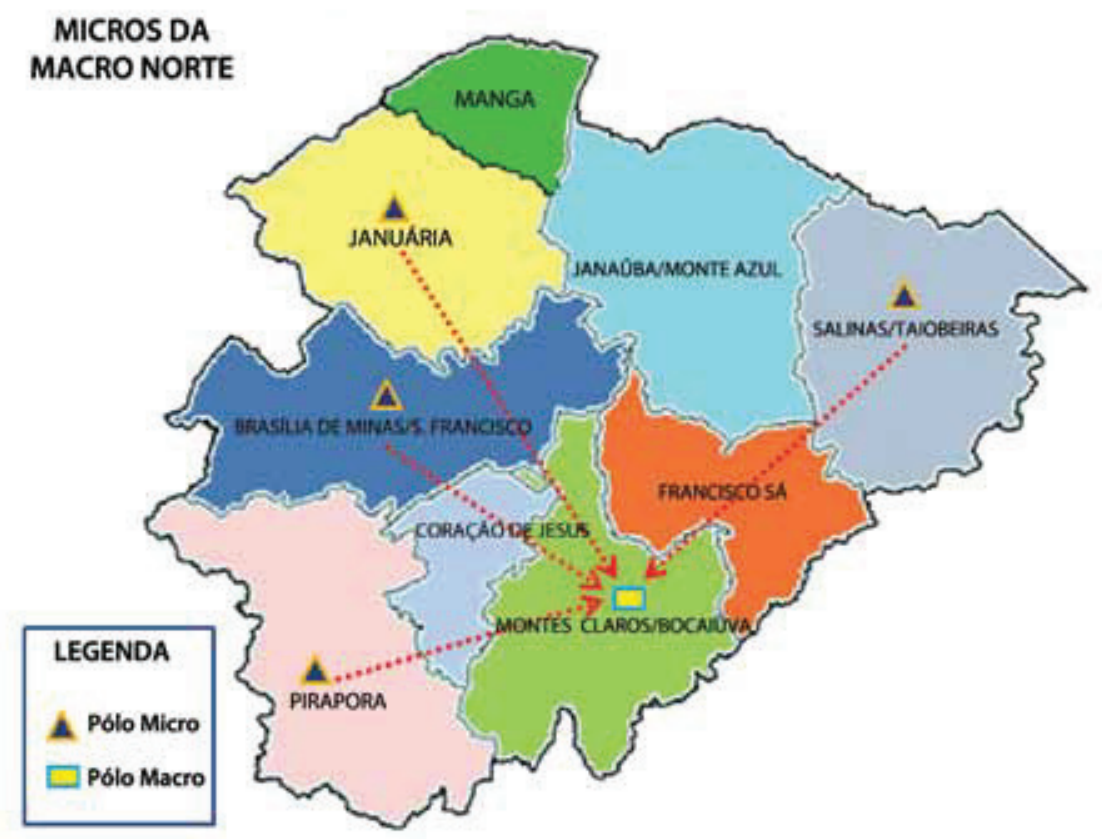

Fonte: PDR/ MG /2009 - Deliberação CIB-SUS/mg n 618 de 09 de dezembro de 2009

De acordo com o Cadastro Nacional de Estabelecimentos de Saúde (CNES, 2009) existem nesta região 208 estabelecimentos de saúde credenciados no SUS com mais de 2.500 leitos hospitalares distribuídos em 40 hospitais. Mais da metade dos leitos está concentrado em estabelecimentos de pequeno porte que apresentam baixa densidade tecnológica, baixa capacitação institucional, por conseqüência precária capacidade resolutiva. Por outro lado, algumas cidades-pólo microrregionais apresentam serviços de suporte com capacidade instalada robusta, e podem ser considerados importantes pólos de atendimento, entre elas, Brasília de Minas, Janaúba, Pirapora e Taiobeiras.

Nota-se que do total dos 86 municípios, a capacidade instalada de serviços de saúde e recursos humanos especializados fica concentrada em menos de cinco municípios. Os municípios citados apresentam proximidade a entroncamentos rodoviários importantes, sendo o segundo entroncamento viário do estado, entretanto a maioria das estradas ainda encontra-se sem asfaltamento o que gera dificuldades, dado as longas distâncias, o deslocamento intermunicipal criando importante grau de complexidade no componente logístico de serviço.

O IDH de MG é de 0,800 (IBGE, estimativa 2005) colocando-o na $10^{a}$ posição em relação aos 27 estados de nosso país. A macrorregião Norte diferencia-se do restante do estado apresentando o indicador próximo ao dos estados mais pobres do Nordeste do Brasil (Alagoas com 0,677), sendo a média da macrorregião de 0,691 (PNUD, 2000). Dado a enorme dimensão territorial e as vicissitudes sócio-demográficas com vigência de baixo índice de desenvolvimento humano, impõem aos agentes públicos grandes desafios na implantação de políticas setoriais, sendo fundamental para a equalização de todas essas desigualdades regionais, que os processos políticos decisórios considerem como diretriz fundamental o princípio da equidade. 


\subsubsection{Situação de Saúde}

De acordo com o estudo de Carga Global de Doenças (Murray e Lopez, 1996), as condições de doenças foram agregadas em três grandes grupos de eventos: Grupo I - Doenças Infecciosas e Parasitárias, causas maternas e perinatais e deficiências nutricionais; Grupo II - Doenças Não Transmissíveis; Grupo III - Causas externas de lesões e ferimentos. Estes, por sua vez, foram subdivididos em 21 categorias mais amplas, e, por conseguinte, desagregadas em mais de 116 eventos específicos.

Para essa região foram perdidos 149.717 anos de vida devido à morte prematura, sendo que $59,0 \%$ foram decorrentes das mortes ocorridas no sexo masculino, quando comparado ao feminino $(P<0,001)$. Os anos de vida perdidos pela macrorregião correspondem a $8,7 \%$ de todos aos anos de vida perdidos no estado de MG. Com exceção das faixas etárias de 1 a 4 anos e 80 anos de mais, em todas as demais observa-se que os homens morrem mais precocemente que as mulheres $(P<0,001)$.

Em relação aos grandes grupos de eventos, nota-se uma redução dos eventos do grupo I à medida que a idade avança, o comportamento oposto pode ser observado nos eventos relacionados ao grupo II. Não se observa grandes diferenças entre os sexos nas faixas etárias analisadas para os eventos do grupo l e II. Para os eventos do grupo III, observa-se que há uma concentração desses eventos em pessoas mais jovens, especificamente em idade economicamente produtiva. Referente aos eventos do grupo III observase que, em ambos os sexos, há uma concentração dos anos perdidos na faixa etária entre 15 e 59 anos.

Nos resultados expostos observou-se a necessidade de formulação de macro políticas que visem à redução das doenças e agravos não transmissíveis, uma vez que são responsáveis por grande dos anos de vida perdidos devido à morte prematura na macrorregião Norte.

Nesse sentido, o estudo de carga de Doença desenvolvido no estado de MG contribui efetivamente na definição de prioridades nos serviços de saúde, por meio da redefinição das áreas de prestação de serviços, do estabelecimento de parâmetros para a programação em saúde, além do mapeamento das necessidades de saúde nos diferentes níveis de assistência e, por fim, na realocação de recursos financeiros.

A resposta social a vigência de tripla carga de doenças é a organização em redes de atenção. Para Mendes, a transição demográfica e epidemiológica resultante do envelhecimento e do aumento da expectativa de vida significa crescente incremento relativo das condições crônicas. A crise contemporânea dos sistemas de saúde caracteriza-se pela organização da atenção em sistemas fragmentados voltados para a atenção às condições agudas, apesar da prevalência de condições crônicas, e pela estrutura hierárquica e sem comunicação fluida entre os diferentes níveis de atenção.

Assim, ações do governo de Minas no âmbito da saúde se distribuem em várias Áreas de Resultado, mas concentram-se mais fortemente na área denominada Vida Saudável, que tem os objetivos estratégicos cada qual com suas respectivas metas de curto, médio e longo prazo: aumento da eficiência alocativa do sistema de atenção à saúde; Saúde em Casa - programa de fortalecimento da atenção primária à saúde; Regionalização da Atenção à Saúde - Programa de Aperfeiçoamento e Melhoria da Qualidade dos Hospitais do SUS - PRO-HOSP - programa de revitalização do parque hospitalar do estado, estimulando os hospitais socialmente necessários a desenvolverem uma política de qualidade; - Rede de Atenção às Urgências e às Emergências - Vida no Vale - COPANOR - programa que visa melhorar a qualidade de vida e promover condições para a prosperidade dos 92 municípios pertencente às bacias hidrográficas dos Rios Jequitinhonha, Mucuri e Leste.

\section{EXPERIÊNCIA EXITOSA - IMPLANTAÇÃO DE LEITOS DE UTI NA MACRORREGIONAL NORTE}

Neste estudo, nos deteremos ao projeto de implementação de UTI nos hospitais selecionados das Microrregiões de Taiobeiras, Pirapora, Brasília de Minas e Janaúba.

A organização das UTI tem se tornado um desafio para os gestores públicos da saúde. Há em todos os países aumento constante na demanda por serviços de UTI conseqüente pressão sobre as estruturas hospitalares e profissionais que atuam diretamente nestes serviços. As UTI também podem ser consideradas como a principal causa de insatisfação da população que utiliza o sistema de serviços de saúde.

Existe consenso de que sempre haverá uma demanda por serviços maior que a necessidade, e que, o aumento da oferta sempre acarreta em aumento da demanda, criando-se assim um sistema de difícil equilíbrio. 
Além disto, a sociedade moderna, cuja demografia sofreu alterações consideráveis, como também as mudanças no perfil de consumo, no acesso à informação e no constante deslocamento populacional, alteraram o fluxo de procura dos serviços de saúde, exigindo nova organização do processo econômico e do perfil de resposta. A solução de gestão tem sido a racionalização da oferta associada a estratégias regulatórias.

A imensa prevalência das condições crônicas e a vigência de tripla carga de doenças nessa região têm implicado a nós gestores e profissionais de saúde na necessária reengenharia na organização e no modo de atuar dos sistemas de saúde.

As estratégias de enfrentamento destes problemas nos pôs condizente na implementação das políticas adotadas na SES/MG, ou seja, no estabelecimento de redes de serviços de saúde com todos os seus componentes.

Há uma grande tendência no SUS de regionalizar os serviços visando às premissas da escala e racionalização e associando o desenvolvimento de processos de gestão da clínica. Neste modelo que utilizamos é visar primordialmente a atenção primária diferenciada que irá estruturar as redes integradas de serviços de saúde.

O estudo de carga de doenças que acompanhamos realizados pela SES/MG compreendendo o período 2004-2006 evidenciou que no sexo masculino as primeiras quatro causas de mortalidade (doenças isquêmicas do coração, doenças cerebrovasculares, violências e acidentes de trânsito) estão relacionadas aquelas condições em que a resposta do sistema de saúde no evento agudo tem importante dependência de organizações maduras de Urgência e Emergência. Já o estudo do indicador Anos Potenciais de Vida Perdidos (APVP) em maiores de um ano, demonstrou que causas externas e doenças cardiovasculares são responsáveis por mais de $46 \%$ dos anos de vida perdidos. O cenário epidemiológico, portanto, justifica fortemente a prioridade do estado de MG, na organização de Redes de Atenção de UeE.

A Figura 11 representa a evolução dos credenciamentos de leitos de UTI no município de Montes Claros. Em 2001, 20 leitos novos leitos inauguraram o serviço de terapia intensiva no município, seguidos pelo credenciamento de mais 08 leitos em 2006, 10 leitos em 2007, 10 leitos em 2008 e 04 leitos em 2009, somando um total de 52 leitos de UTI adulto, totalmente equipados para atender a população do SUS.

Figura 3: Evolução dos credenciamentos em Portaria Ministerial (PM) dos leitos de UTI adulto em Montes Claros

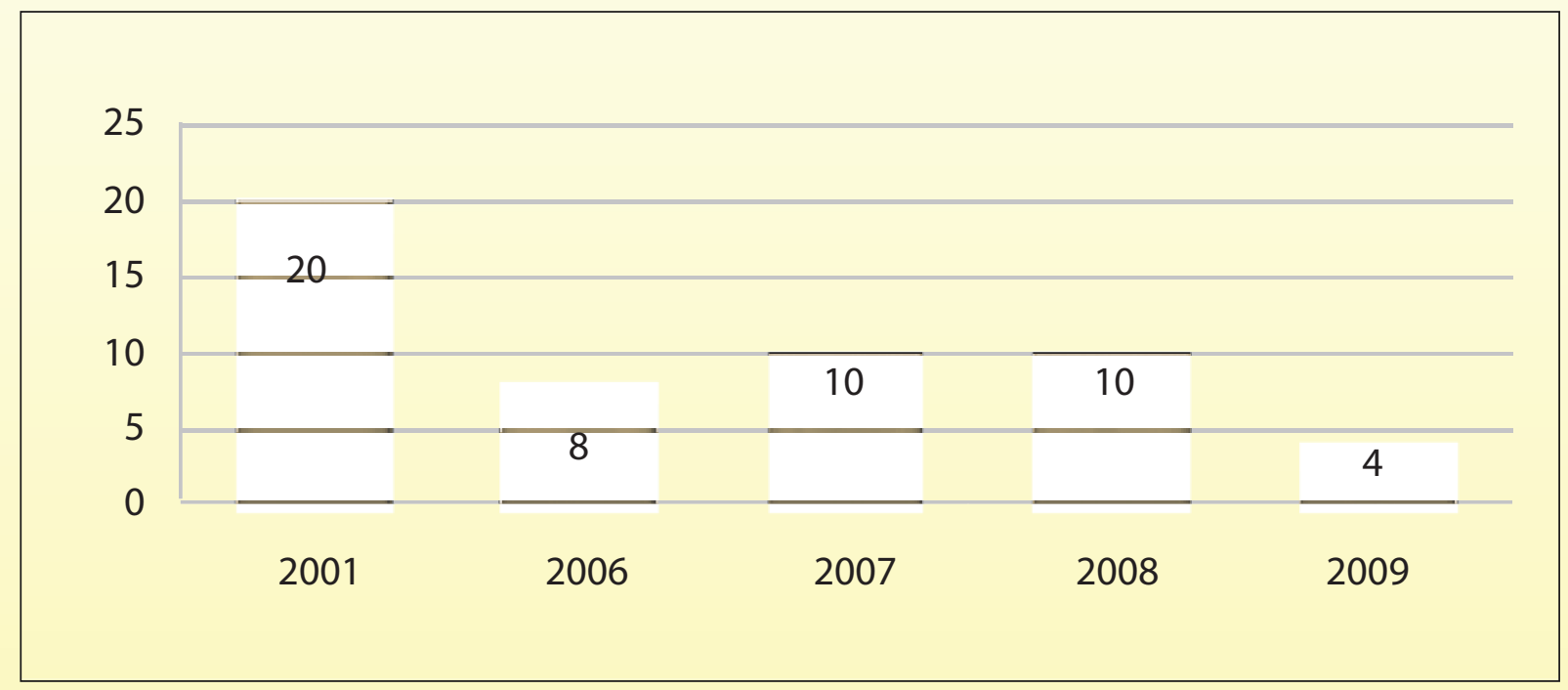

Fonte: SES/MG - Coordenação de UTI - Set. 2010

Visando a crescente ampliação do serviço na Macrorregião Norte, e com o objetivo de descentralizar o serviço de atendimento em terapia intensiva, MG investiu e incentivou a construção, equipagem e o credenciamento de leitos de UTI na Macrorregião, somando um total de 40 novos leitos de UTI, distribuídos entre: Taiobeiras, Pirapora, Brasília de Minas e Janaúba, sendo 10 leitos em cada um destes municípios. Os credenciamentos com publicação de Portaria e liberação de verba pelo Ministério da Saúde (MS) dos 40 leitos de UTI ocorreu nos períodos de: 18 de Dezembro de 2009 (Pirapora e Brasília de Minas) a 09 de Abril de 2010 (Janaúba e Taiobeiras), conforme Figura 12. 
Figura 4: evolução dos credenciamentos em PM dos leitos de UTI Adulto nas demais Microrregiões da Macrorregião Norte.

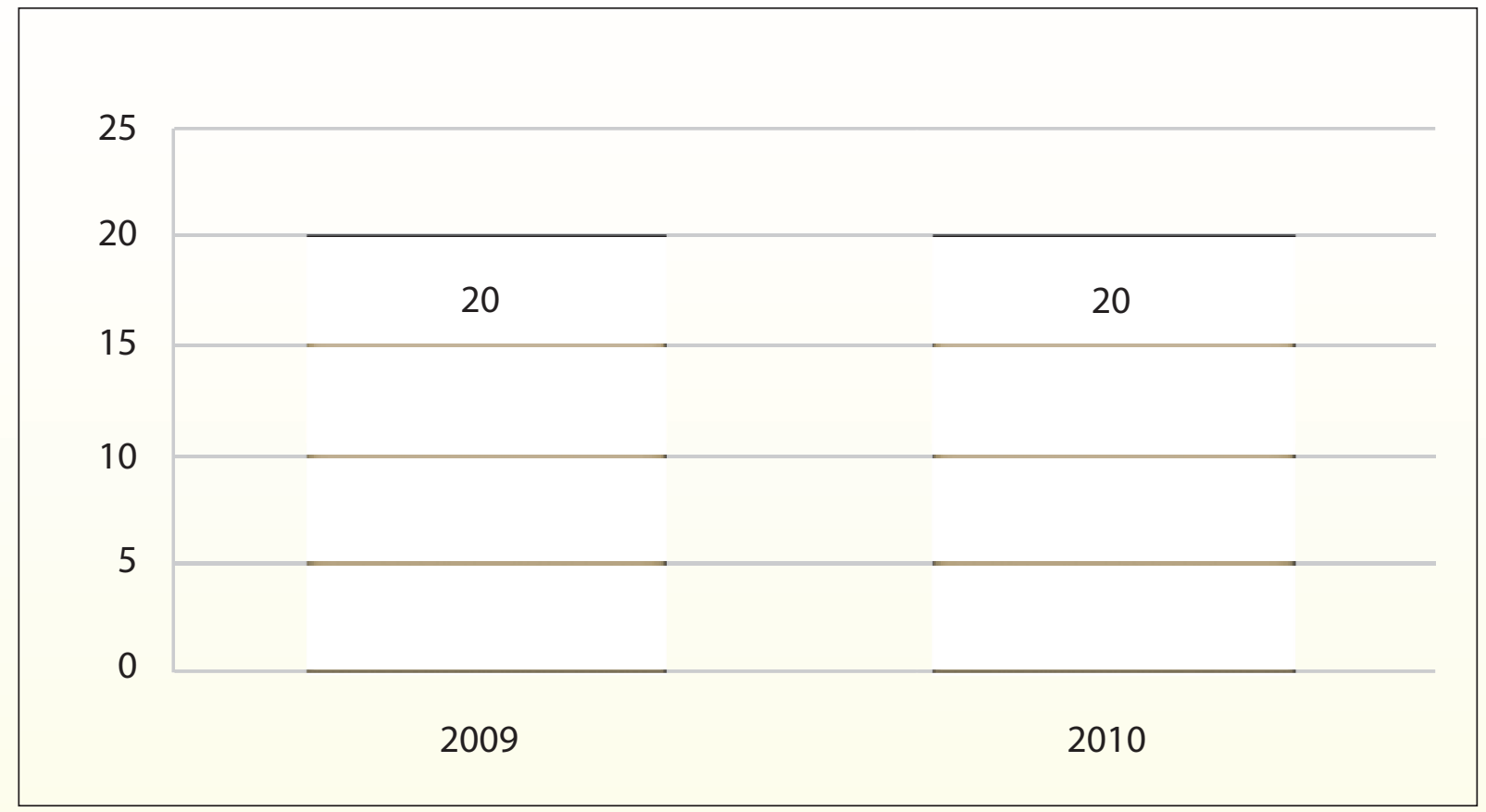

Fonte: SES/MG - Coordenação de UTI - Set. 2010

\section{RESULTADOS ALCANÇADOS}

O parque de leitos de UTI foi ampliado significativamente no total de 40 leitos nos municípios de Brasília de Minas, Janaúba, Pirapora e Taiobeiras, onde foram criadas câmaras técnicas de trauma, cardiologia e AVC e um núcleo de educação permanente implantado. Abaixo mencionaremos os resultados nas Microrregionais estudadas.

\subsection{Microrregião Pirapora - Fundação Dr. Moisés Magalhães Freire}

A Fundação Dr. Moisés Magalhães Freire, reinaugurada em 2008, depois de desabar por causa de fortes chuvas ocorridas em 2005. Hoje, o hospital pode comemorar, além da inauguração de UTI, o fato de não haver pessoas na fila de espera para cirurgias eletivas, um dos pontos mais críticos do SUS. Desde sua reinauguração já foram realizados mais de 100 mil atendimentos.

Foi a primeiro, além de Montes Claros, a dispor de leitos de UTI adulto no Norte de Minas. A Rede de Urgência Norte busca qualificar o atendimento de urgência em toda a região. O investimento do Governo de Minas, de aproximadamente R\$ 2 milhões, em Pirapora, beneficiou diretamente cerca 150 mil moradores da microrregião de Pirapora, e consequentemente todos os 86 municípios do Norte de Minas.

A UTI foi inaugurada em 25 de novembro de 2009 com capacidade de dez leitos para terapia intensiva de adultos e capacidade resolutiva para casos de diversas complexidades. Abre precedente para outras especialidades e serviços. Promove abrangência para toda Macro Norte e outras macros. Proporciona atendimento para os usuários do SUS e de Convênios. Sua equipe é formada por: - oito médicos; - quatro enfermeiros; - três enfermeiros; - vinte técnicos de enfermagem; - uma secretária; - dois funcionários da limpeza. Tendo as comissões: - Comissão de Controle de Infecção Hospitalar - CCIH; - Comissão de Nutrição - NUTRIFEM; - Comissão de Curativos; - Comissão Interna de Prevenção de Acidentes - CIPA.

A ocupação dos leitos de UTI desta Fundação é formada por 58,8\% por pessoa do sexo masculino e 41,2\% por pessoas do sexo feminino, de acordo Figura 5, que demonstra que o sexo masculino está $18 \%$ a mais do que sexo feminino utilizando os leitos de UTI, que poderemos comparar com os dados percentuais de morbidade da Macrorregional Norte já mencionados anteriormente. 
Figura 5: Percentual dos leitos de CTI ocupados por sexo

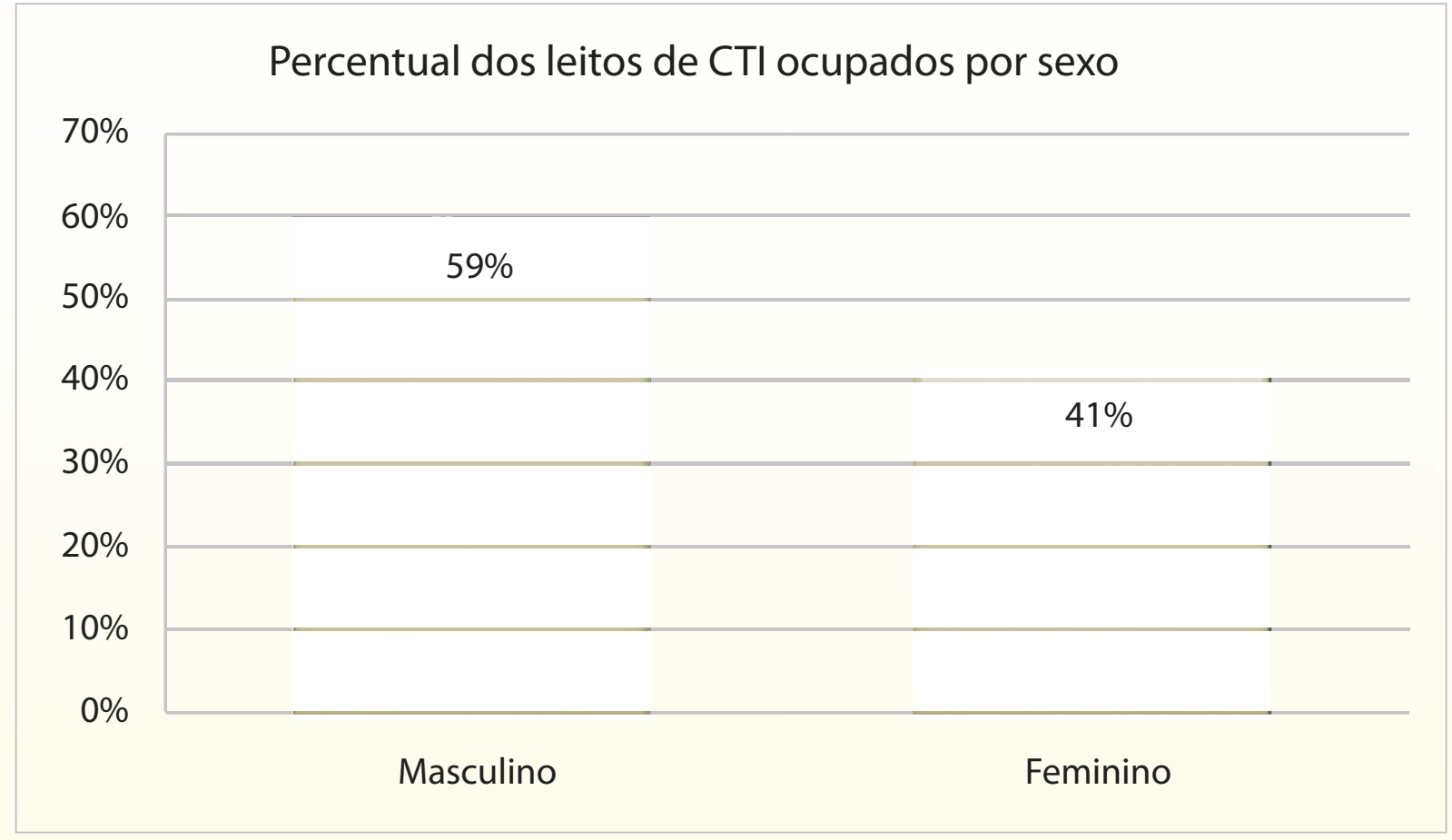

Fonte: Balanço Assistencial - Fundação Dr. Moisés Magalhães Freire

Constata-se também que a idade média da população atendida no CTI - Fundação Dr. Moisés Magalhães Freire é 59,4 anos.

A Fundação pesquisou a procedência dos pacientes que eram atendidos na UTI e constatou que em primeiro lugar a demanda vem do Pronto Socorro, seguido em segundo lugar das Internações, em terceiro lugar Bloco Cirúrgico, depois em quarto lugar do SUS Fácil e por último, em quinto lugar de outros hospitais, Figura 6.

Figura 6: Procedência dos pacientes atendidos na UTI

\section{Procedência dos Pacientes atendidos na UTI}

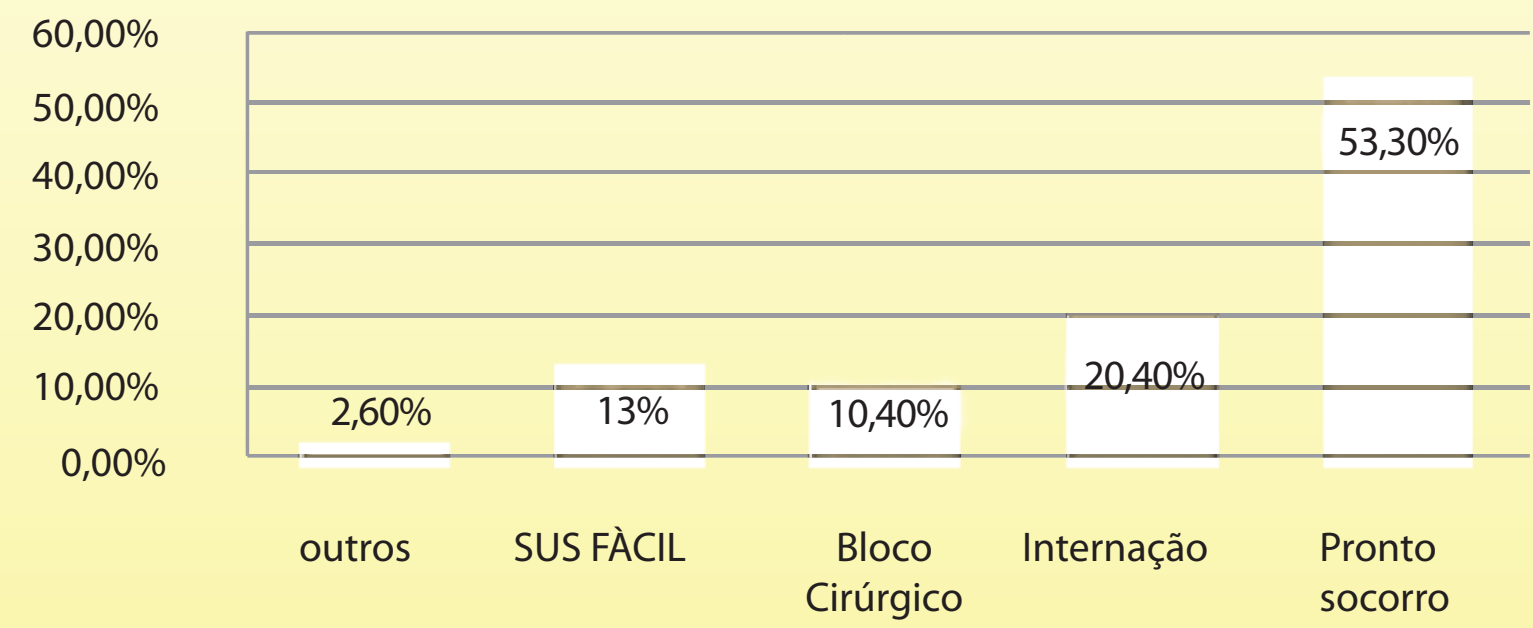

Fonte: Balanço Assistencial - Fundação Dr. Moisés Magalhães Freire 
Nessa pesquisa a Fundação constatou também os agravos que motivaram as internações, em ordem de prevalência mencionamos que os Acidentes Vasculares têm uma incidência significativa - 41,4\%, seguida das infecções respiratórias - 16,7\%, depois doenças do aparelho digestivo - 6,7\%, após constatamos que os problemas neurológicos conjugados com acidentes vasculares encefálicos e os pós-operatórios - 11,1\%, as infecções $-7,1 \%$, em penúltimo lugar os traumas - 4,8\%, e em último lugar as intoxicações $1,1 \%$, como demonstrado na Figura 7.

Figura 7: Percentuais dos agravos que motivaram as internações na UTI Agravos que motivaram as internações na UTI
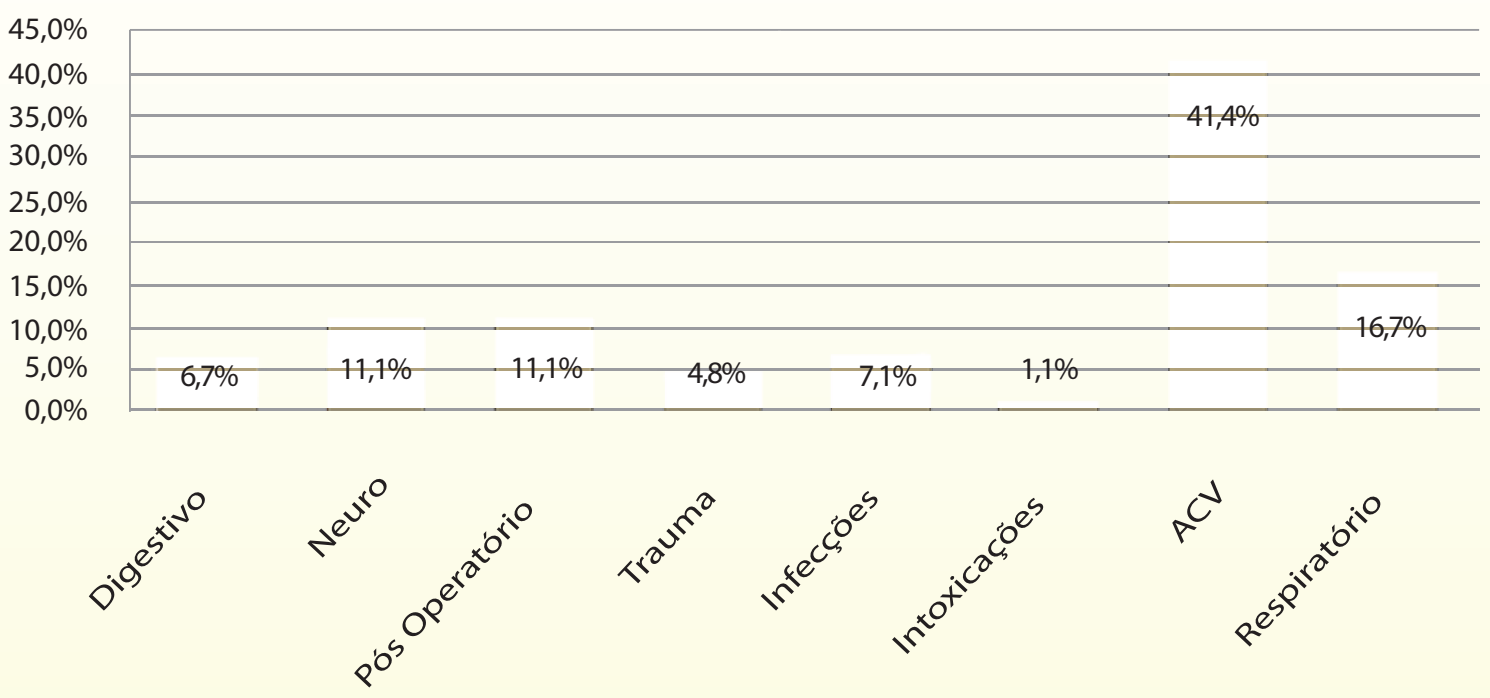

Fonte: Balanço Assistencial - Fundação Dr. Moisés Magalhães Freire

A figura 19 mostra que a incidência de alta da população que ocupa os leitos de UTI é de 56,5\%, maior que as transferências que são de $16,4 \%$, e que os óbitos ocupam $27,1 \%$ infelizmente, pois a meta da Fundação é que esses valores caiam cada vez mais.

Figura 8: Percentuais de altas/transferências/óbitos

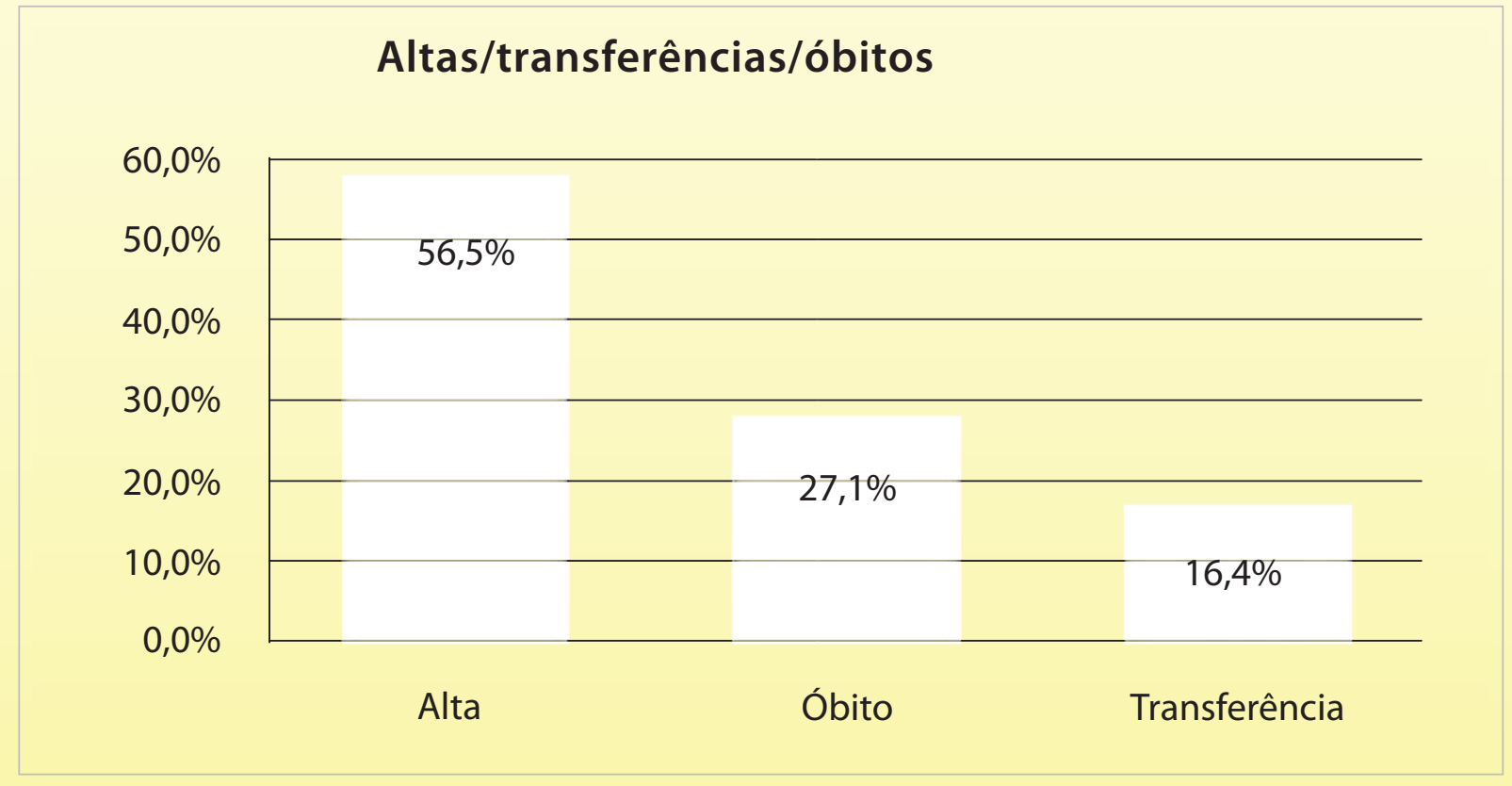

Fonte: Balanço Assistencial - Fundação Dr. Moisés Magalhães Freire 
Constatou-se como mostra a Tabela 1 a média de idade da população que ocupa os leito de UTI, a média total da população é de 59,4 anos, sendo que a média da população que vai a óbito é mais idosa, ou seja, 67,0 anos. E mostra também AVC - 25,4\%, Respiratório - 11,3\%, Digesivo - 11,3\%, Neuro/AVE - 8,4\%, Pós-Operatório, Trauma - 4,2\%, Infecções - 32,4\%, Intoxicações - 1,4\%.

Tabela 1: média de idade da população de UTI - motivos dos óbitos

\begin{tabular}{c|c}
\hline População UTI & Média de idade - anos \\
\hline Total & 59,4 \\
Óbitos & 67,0 \\
\hline
\end{tabular}

Fonte: Balanço Assistencial - Fundação Dr. Moisés Magalhães Freire

Os relatados acima são mostrados em gráfico setorial para melhor visualização, na Figura 9 a abrangência dos agravos que vão a óbito evidenciando claramente que as infecções são as maiores causas de morte, seguidas das doenças cardiovasculares.

Figura 9: Motivos dos óbitos

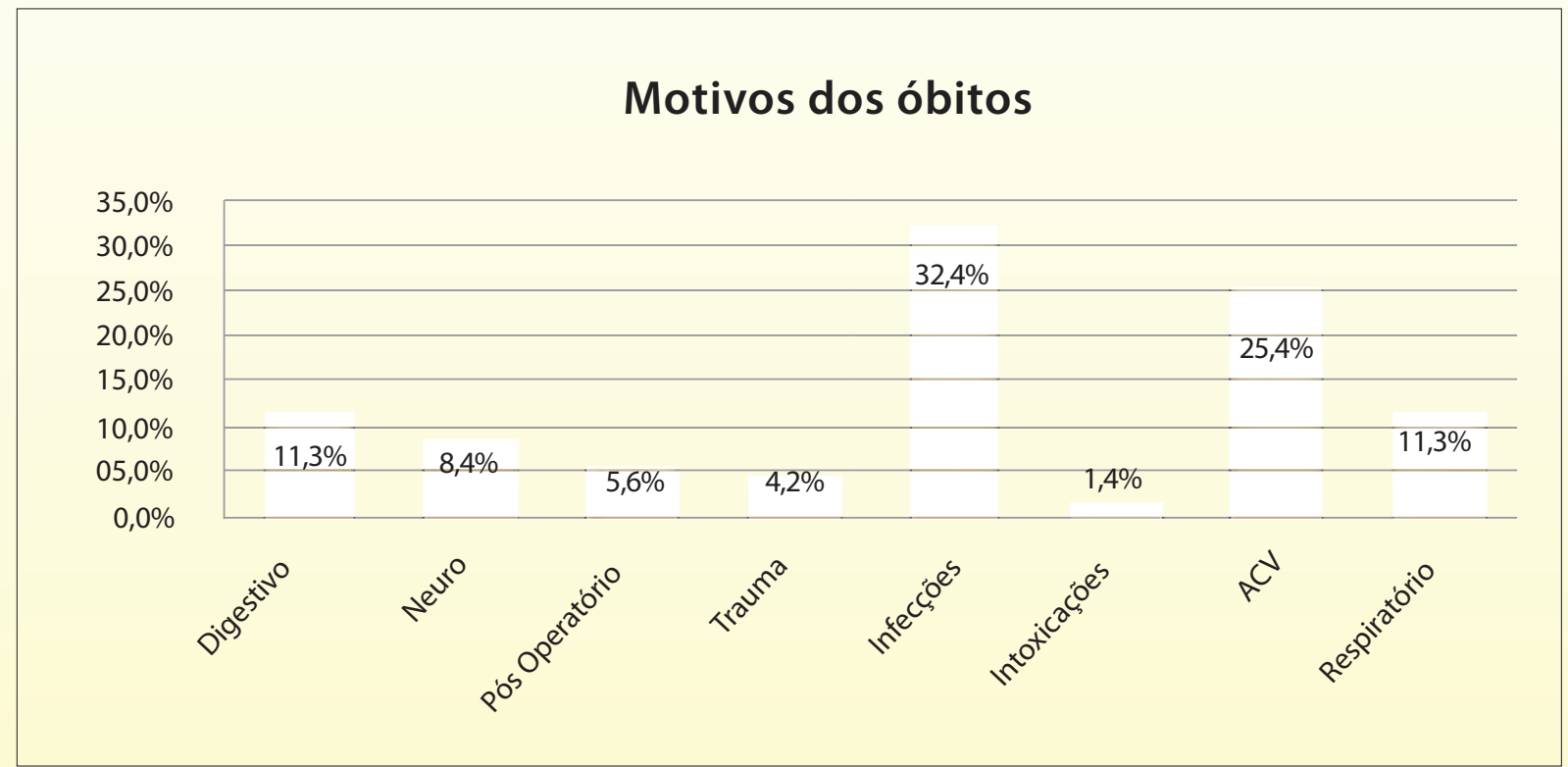

Fonte: Balanço Assistencial - Fundação Dr. Moisés Magalhães Freire

A UTI dessa Fundação apresenta os dados, Tabela 2, mostrando que dos 285 pacientes atendidos, 75 não resistem e vão infelizmente a óbito, perfazendo uma taxa de 26,3\%, ou seja, se salva 73,7\%, assim, essa instituição deseja cair esse percentual cada vez mais até atingir o ideal salvar $100 \%$ das pessoas por ela atendidas.

Tabela 2: número de pacientes atendidos - total de óbitos

\begin{tabular}{|c|c|c|}
\hline \multicolumn{2}{|c|}{ Número de pacientes atendidos } & \multirow{2}{*}{ Taxa de Óbito $-\%$} \\
\hline recuperados & óbitos & 26,3 \\
\hline 285 & 75 & 26,3 \\
\hline
\end{tabular}

Fonte: Balanço Assistencial - Fundação Dr. Moisés Magalhães Freire 
A relação profissional e fraterna entre paciente e equipe de saúde é primordial para o tratamento, levando-se em conta também uma equipe profissional unida, comprometida e capacitada que proporciona aos seus pacientes uma melhor recuperação dos pacientes, e assim para que todos alcancem cada vez mais uma vida saudável.

\subsection{Microrregiões de Brasília de Minas, Taiobeiras e Janaúda}

No ano de 2009 foram inaugurados 40 novos leitos de UTI disponíveis na Rede de Urgência, Norte, sendo10 leitos em Brasília de Minas, 10 leitos em Taiobeiras e outros 10 em Janaúba. Capacitados mais de 2.500 profissionais desde a sua implantação. O Complexo Regulador e o SAMU regionalizado são pioneiros no Brasil e estão no nível de países do primeiro mundo. O salto de qualidade no atendimento e a evolução da capacidade técnica são visíveis.

\subsection{Microrregiões de Pirapora, Brasília de Minas, Taiobeiras e Janaúba - Investimento de Recursos Financeiros}

Os investimentos de recursos financeiros aplicados nesses municípios da Macrorregião Norte, Tabela 3 , Pirapora, Brasília de Minas, Pirapora, Taiobeiras e Janaúba.

Tabela 3: Investimento de Recursos Financeiros - Brasília de Minas - Pirapora e Taiobeiras

\begin{tabular}{|c|c|}
\hline CUSTEIO & $\mathrm{R} \$$ \\
\hline CUSTEIO (convênio 568/08) & 11.374.971,07 \\
\hline \multicolumn{2}{|c|}{ REDE } \\
\hline Rede Resposta & $16.000 .000,00$ \\
\hline \multicolumn{2}{|c|}{ UTI } \\
\hline \multicolumn{2}{|c|}{ Investimento } \\
\hline UTI Pirapora & $292.676,40$ \\
\hline UTI Janaúba & $330.916,40$ \\
\hline UTI Brasília de Minas & $302.656,40$ \\
\hline UTI Taiobeiras & $318.176,40$ \\
\hline Construção Pronto Socorro Brasília de Minas & $505.000,00$ \\
\hline Construção Pronto Socorro Taiobeiras & $495.000,00$ \\
\hline \multicolumn{2}{|c|}{ TOTAL } \\
\hline Geral & $32.883 .526,67$ \\
\hline Investimentos & $5.508 .555,60$ \\
\hline Custeio & $27.374 .971,07$ \\
\hline
\end{tabular}

Fonte: Coordenadoria de UTI - SES/MG - Set. 2010 


\subsection{Estruturação da Rede UeE na Macrorregião Norte}

A estruturação da Rede de UeE na Macrorregião Norte com foco nos leitos de UTI é fruto de forte vontade política, capacidade técnica e operacional. Tem como objetivo descentralizar o atendimento. O cidadão deve ser atendido pela pessoa certa, no tempo certo e no lugar certo. Essa experiência êxitos é pioneira, nos próximos anos, vai apresentar indicadores de redução da mortalidade muito importantes.

A Rede de UeE nessa Macrorregião busca qualificar o atendimento de urgência em toda a região. $O$ investimento do Governo de Minas, de aproximadamente R\$ 2 milhões, em Pirapora, beneficiou diretamente cerca 150 mil moradores da microrregião de Pirapora, e consequentemente todos os 86 municípios do Norte de Minas.

A Macrorregião Norte de Minas foi à primeira contar com Serviço de Atendimento Móvel de Urgência (SAMU) Regional. Todos os 86 municípios integrantes da Macro Norte passam a contar com o serviço e, para colocá-lo em prática, tem à disposição 38 ambulâncias básicas, 06 avançadas e um helicóptero. Para tanto, foram investidos cerca de R\$ 50 milhões.

Nos seis primeiros meses, o SAMU recebeu cerca de 21.839 chamados, por meio do Fone: 192 . São cerca de 300 ligações por dia, que geram cerca de 100 saídas de ambulâncias para atendimento ou transferência de pacientes de uma unidade hospitalar para a outra que contém UTI.

O Complexo Regulador e o SAMU regionalizado são pioneiros no Brasil e estão no nível de países do primeiro mundo. O salto de qualidade no atendimento e a evolução da capacidade técnica são visíveis.

O Complexo Regulador da Macrorregião Norte conta com quase 600 funcionários, sendo 70 médicos reguladores e intervencionistas, 31 enfermeiros, 210 Técnicos de enfermagem, 215 condutores/socorristas, 30 administrativos e 08 rádio-operadores, distribuídos nas bases da região. No Complexo funcionam a Central de Urgência e Emergência do SAMU Regional e a Central de Regulação Assistencial.

\section{CONSIDERAÇÕES FINAIS}

É com satisfação que mencionamos o depoimento do médico suíço, Dr. Gian Gessam Meger, em visita a Macrorregional Norte em 2009, que já participou de mais de 30 missões pelo mundo com a Cruz Vermelha Internacional e com Organização Mundial de Saúde, ficou surpreso com a estrutura montada no Norte de Minas: "a estrutura física está no mesmo nível dos países europeus. É importante também que os profissionais sejam capacitados constantemente, as evoluções da medicina são muito rápidas e os profissionais devem sempre estar atualizados", afirmou.

Avançar cada vez no aperfeiçoamento do Planejamento Estratégico nesse estudo foi o nosso grande aprendizado utilizando o caminho da ordenação de prioridades, a adoção das correções e aperfeiçoamentos no momento certo, assim constatamos mais uma vez que planejar é preciso. Esse foco nos conduziu em nossa missão de SALVAR VIDAS e amenizar o sofrimento das pessoas proporcionando VIDA SAUDÁVEL para toda população atendida nas UTI dos hospitais da Rede UeE.

Unidos nesse propósito em conjunto com as demais Secretarias de Estado de Minas Gerais poderemos melhorar cada vez mais os Indicadores dessa Região Norte nas áreas de Saúde, Social, Segurança, Educação, Saneamento Básico, dentre outras. Assim conseguiremos trabalhar para aliviar as condições em todas as instâncias desse povo tão carente.

Assim, imbuídos dessa visão poderemos trabalhar em prol dessa população proporcionando melhores condições de vida.

Neste contexto estamos gratos aos prefeitos, gestores, profissionais, e sua população dessa região por nos facilitar sermos protagonistas dessa experiência exitosa que ora apresentamos.

Parabenizamos esse povo pelo orgulho de sua terra, perseverança, alegria e de acreditar que é possível ter serviços de qualidade em saúde apesar de indicadores desfavoráveis, que a iniqüidade não é por si só uma incapacidade. 


\section{REFERÊNCIAS}

MARANHÃO, E. Avaliação por Carga de Doença. Disponível em: <http:www4.ensp.fiocruz.br/ biblioteca/dados/txt_153961933.ppt. > Acesso em: 12 de set.2010.

BRASIL. MINISTÉRIO DA SAÚDE. Coordenação Geral de Informações e Análise Epidemiológica. Análise da Situação de Saúde. Brasília, DF, 2004.

CARNEIRO, M. F. B. Organização Espacial de Montes Claros e a Região Norte de Minas Gerais. Dissertação (Mestrado em Geografia). Faculdade de Filosofia, Letras e Ciências Humanas. Universidade de São Paulo (USP). São Paulo, 2002.

DATASUS. Sistema de informações em saúde. Informações demográficas e socioeconômicas: população residente. Disponível em: <http:www.tabnet.datasus.gov.br>. Acesso em: 8 de ago. de 2008.

FRANÇA, I. S. As novas centralidades de uma cidade média: o exemplo de Montes Claros no Norte de Minas Gerais. 2007. 240 f. Dissertação (Mestrado em Geografia), Universidade Federal de Uberlândia (UFU), Minas Gerais, 2007.

GUIMARÃES, B. T.; Planejar é preciso, Curso Gerenciamento para Gestores do Governo de Minas Gerais, Fundação Dom Cabral, 2009.

INSTITUTO BRASILEIRO DE GEOGRAFIA E ESTATÍ́STICA. IBGE. Censo Demográfico de Montes Claros, Minas Gerais. 1950-2000.

Demográfico: Minas Gerais. Rio de Janeiro: IBGE; 1980-1991 e 2000. Acesso em: agosto de 2008. Disponível em: http://www.ibge.gov.br.

LEITE, R. F. C. Norte de Minas e Montes Claros: o significado do ensino superior na (re) configuração da rede urbana regional. 2003. 191p. Dissertação (Mestrado em Geografia) Universidade Federal de Uberlândia, Minas Gerais, 2003.

MARQUES, A. J. M. O Choque de Gestão na Saúde em Minas Gerais. Belo Horizonte, Ed. Autêntica, 2009. p. 29 - 44.

NOTíCIAS. Região Sudeste. Pirapora recebe 10 leitos de UTI em novembro de 2009. Disponível em: <http: www.brasilsus.com.br/.../101482-pirapora-recebe-10-leitos-de-uti.html>. Acesso em: 26 de dez. 2009.

WORLD HEALTH ORGANIZATION - WHO. The world health report 2000: health systems, improving performance. Geneva, WHO, 2000. 